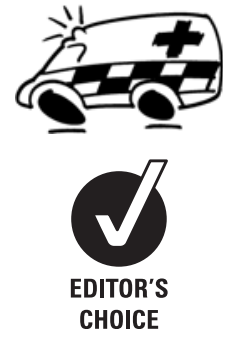

${ }^{1}$ Faculty of Health and Social Care Sciences, Kingston University and St George's, University of London, London, UK ${ }^{2}$ Bentleigh Bayside Community Health, Melbourne, Australia

${ }^{3}$ Centre for Health Information, Research and Evaluation, Swansea University, Swansea, UK

${ }^{4}$ Prince of Wales Hospital and Prince of Wales Medical Research Institute, University of New South Wales, Sydney, Australia

5London Ambulance Service NHS Trust, London, UK ${ }^{6}$ Royal Free Hampstead NHS Trust, London, UK

\section{Correspondence to}

Mary Halter, Faculty of Health and Social Care Sciences, Kingston University and St George's, University of London, Grosvenor Wing, London SW17 ORE, UK ;

m.halter@sgul.kingston.ac.uk

Accepted 22 December 2009 Published Online First 14 May 2010

\title{
Complexity of the decision-making process of ambulance staff for assessment and referral of older people who have fallen: a qualitative study
}

\author{
Mary Halter, ${ }^{1}$ Susan Vernon, ${ }^{2}$ Helen Snooks, ${ }^{3}$ Alison Porter, ${ }^{3}$ Jacqueline Close, ${ }^{4}$ \\ Fionna Moore, ${ }^{5}$ Simon Porsz ${ }^{6}$
}

\begin{abstract}
Background Older people who fall commonly present to the emergency ambulance service, and approximately $40 \%$ are not conveyed to the emergency department (ED), despite an historic lack of formal training for such decisions. This study aimed to understand the decisionmaking processes of emergency ambulance staff with older people who have fallen.

Methods During 2005 ambulance staff in London tested a clinical assessment tool for use with the older person who had fallen. Documented use of the tool was low. Following the trial, 12 staff participated in semistructured interviews. Interviews were recorded and transcribed. Thematic analysis was carried out.

Results The interviews revealed a similar assessment and decision-making process among participants:

- Prearrival: forming an early opinion from information from the emergency call.

- Initial contact: assessing the need for any immediate action and establishing a rapport.

- Continuing assessment: gathering and assimilating medical and social information.

- Making a conveyance decision: negotiation, referral and professional defence, using professional experience and instinct.
\end{abstract}

Conclusions An assessment process was described that highlights the complexity of making decisions about whether or not to convey older people who fall and present to the emergency ambulance service, and a predominance of informal decision-making processes. The need for support for ambulance staff in this area was highlighted, generating a significant challenge to those with education roles in the ambulance service. Further research is needed to look at how new care pathways, which offer an alternative to the ED may influence decision making around non-conveyance.

\section{BACKGROUND}

Falls in older people are recognised as an issue internationally, ${ }^{12}$ and are associated with high human (social isolation and functional deterioration) ${ }^{3} 4$ and organisational (3\% of the total National Health Service expenditure in the UK) costs. ${ }^{5}$ The prevention of falls in older people is a priority area within the UK. ${ }^{6}{ }^{7}$ Older people commonly present to the emergency ambulance service (999) following a fall-approximately 60000 attendances, or $8 \%$ of the total emergency ambulance response each year in London (UK), and $7.5 \%$ of the emergency workload in an urban US service. $^{8}$ At the time of this study, ambulance staff (other than emergency care practitioners (ECPs)) in London were not formally trained to make decisions regarding the appropriateness of conveyance to the emergency department (ED) and treatment guidelines indicated that all patients should be conveyed unless the patient refused. (An ECP is a new role working in emergency ambulance and other healthcare settings in the UK. Those undertaking the role are usually paramedics or nurses who undertake further education to enable them to assess and treat patients, with an aim of avoiding attendance at an ED or admission to hospital where possible.) Although the numbers of ECPs remain very small, the rate of non-conveyance to the $\mathrm{ED}$ is high for older people who fall-approximately $40 \%$ in London ${ }^{9}$ and elsewhere in the UK ${ }^{10} 11$ and in the US. ${ }^{8}$ Those who are not conveyed have been found to be a group at high risk of further falls. ${ }^{9} 12$

No literature has been identified that specifically examines the process of decision making by ambulance staff in relation to older people who fall. However, decision making regarding conveyance in general has been found to be a complex and negotiated process ${ }^{13}$ dependent upon a number of factors including the experience and confidence of ambulance staff, time during a shift, location, the wishes of the patient, presence of carers, appearance of the person's accommodation, waiting times at the local $\mathrm{ED}$ and prior knowledge of the patient, ${ }^{10}{ }^{14}$ carried out in the context of anxiety about whether a nonconveyance decision will be supported within the ambulance service. ${ }^{10} 15$

Against this background, a study was set up to develop, implement and evaluate an educational package and 'clinical assessment tool' (CAT) to support ambulance staff to make consistent and formalised decisions concerning the conveyance of older people who have fallen. The CAT is a flowchart, with accompanying definitions, guiding the user through an assessment to decide whether the patient is safe to remain at home rather than be conveyed to the ED (see figure 1). The intervention, definitions used, participants, methods and key findings from the quantitative aspect of the study are summarised in table 1 and reported in full elsewhere. $^{16}$

The qualitative strand of the study took an interpretative phenomenological approach to uncover the lived experiences of the ambulance staff trained in the use of a clinical assessment tool for the study, addressing the question: "what are the processes used by ambulance staff in the assessment of older people 
Figure 1 Assessment and referral tool tested during the study, prior to interviews.
London Ambulance Service NHS Trust

Research Project: Fit to be left?

This tool is ONLY for use by LAS staff trained as part of the research project 2003

LAS HELPING ELDERLY FALLERS ASSESSMENT TOOL

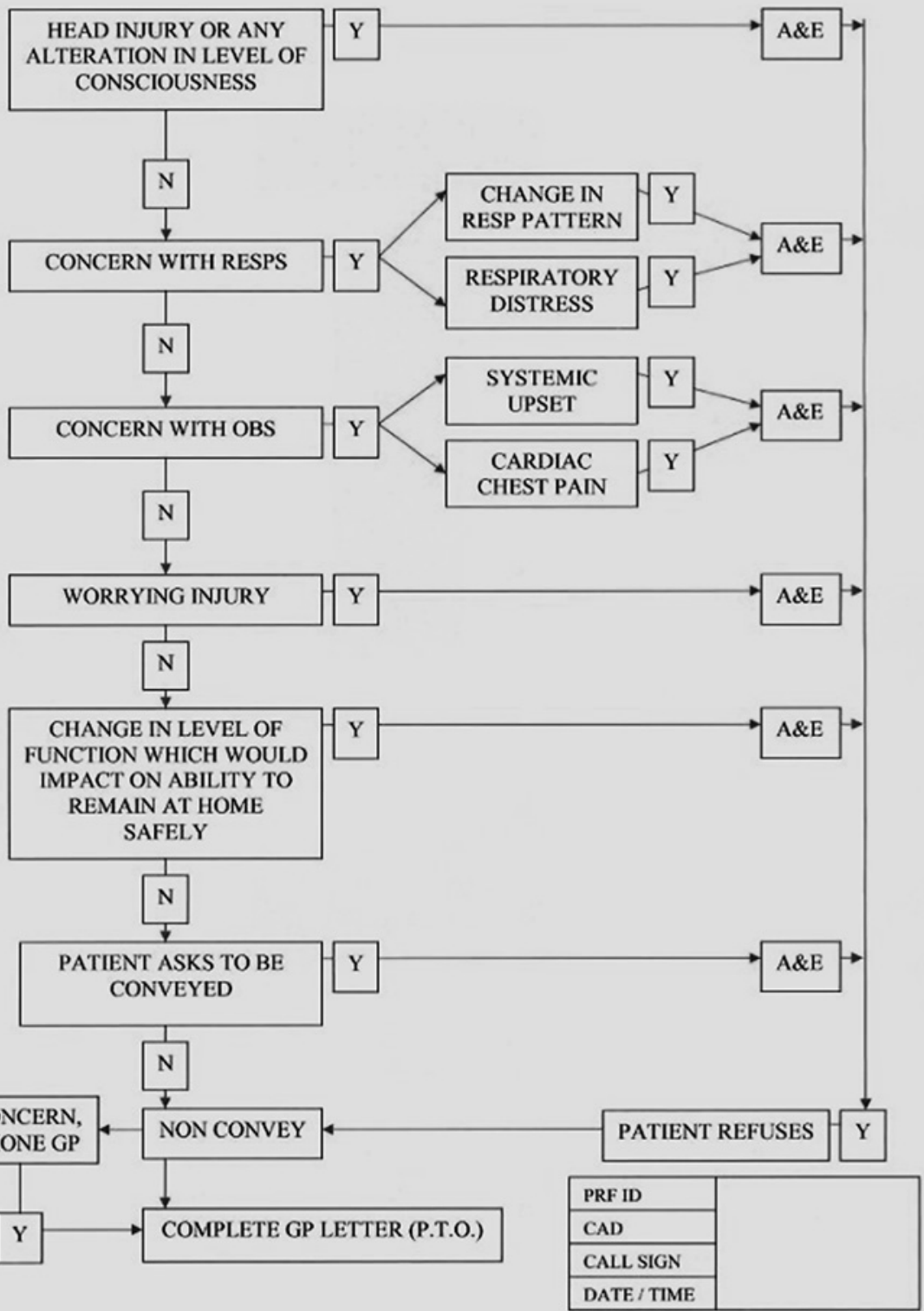

who have fallen?' The purpose of addressing this question was to gain a greater understanding of these practices following implementation of the CAT, in the context of low usage rates identified during the study.

\section{METHODS}

\section{Study setting}

London Ambulance Service NHS Trust provides an emergency ambulance response to almost 1 million patients each year. The majority of responses are provided by traditional ambulances, each usually staffed by one emergency medical technician (EMT) and one paramedic. These staff are guided to perform baseline observations of blood pressure, pulse, respiration, blood sugar and consciousness level (Glasgow Coma Scale) as part of a 'primary survey' of every patient, prior to commencing a more specific 'secondary survey' and treatment appropriate to the patient's presenting issue. Conveyance to the ED has traditionally been recommended for all patients by Ambulance Service guidelines.

Research ethics approval for the study was granted by the Metropolitan Multi Centre Research Ethics Committee.

\section{Data generation}

A semistructured interview was used to collect data. The interview guide asked staff to describe their processes of decision making, after their involvement in the 6-month implementation period of the study CAT. Interviews were recorded and transcribed. 
Table 1 A summary of the participants, methods and key findings of the intervention study to test the clinical assessment tool (CAT)

Characteristic

\section{Findings/values}

Study period

Development of CAT

Content of the CAT

CAT study participants

Preparation of participants

Nature of participation

Definitions

Evaluation of the CAT: tool use

Evaluation of the CAT: impact on 'adverse events'

2003-2004

Team of clinicians (community, ambulance and acute backgrounds) and patient representatives.

The CAT was designed to shadow the established format of prehospital guidelines but differed in providing a fallsspecific assessment guide, prompts to conveyance or non-conveyance to the $E D$ and a route of referral to the patient's GP.

Assessment of any 'change in level of function which would impact on the ability to remain at home safely', with function defined as physical, mental and social.

All EMTs and paramedics $(n=213)$ based in eight ambulance stations (in four groups) in London: $61.1 \%$ male, median length of service 2 years.

2-h training session on the causes of falls, assessment of the older person who has fallen, use of the CAT and research methods.

Completion of a CAT for every patient meeting the definitions below for a 6-month patient recruitment period. Use of the CAT was encouraged during training and by the study researchers, but staff were not held to account for non-

A fall: 'Inadvertently coming to rest on the ground or other lower level with or without loss of consciousness and other than as a consequence of sudden onset of paralysis, epileptic seizure or overwhelming external force. An older person $=$ aged 65 and over Use in practice varied between staff members, with $22.8 \%$ of the participating staff completing the tool in all appropriate cases they attended, $47.8 \%$ in some and $29.4 \%$ in no cases.

A statistically significant reduction in adverse events (reattendance by the ambulance service or to the $\mathrm{ED}$, or death) during the study period was found when compared with baseline data ${ }^{9}(34.6 \%$ intervention period vs $49.1 \%$ baseline period; $\chi^{2} 11.208, \mathrm{p}=0.001$ ).

\section{Participants}

A convenience sample of 12 ambulance staff was recruited. The researcher invited ambulance staff to participate in person at their ambulance station during routine weekly research visits. The first three staff members who consented at each of the four ambulance station groups were interviewed. The participants were 7 women and 5 men ( 1 paramedic and 11 EMTs) with a mean of 3.5 years and a median of 1 year's length of service, who all completed an assessment tool for between $0 \%$ and $88 \%$ of the eligible cases they had attended in the intervention period.

\section{Data analysis}

A thematic analysis was carried out. Interview transcripts were read extensively and a thematic index (of the overarching concepts of definition of fall, establishing a cause, instinct, environment, physical issues, mobility, willingness to go to hospital, other professionals, social aspects and miscellaneous) developed after discussion between two researchers. Two tran- scripts were independently indexed by two researchers and then compared to ensure transparency. ${ }^{17}$ Summaries and direct quotes from the transcripts were placed into a table depicting the thematic index. During this analysis a 'process' of decision making emerged that contained the above thematic concepts, and the data were reindexed and a narrative account of this process developed, supported by extracts from participants to capture the richness of categories.

\section{RESULTS}

Analysis of the interviewees' transcripts revealed a similar overall approach to assessment, showing a broadly sequential pathway through the decision-making process with an older person who has fallen. This process has been split into four stages: prearrival, initial contact, continuing assessment and making a conveyance decision.

\section{Prearrival: using background information to form an early opinion}

The assessment and decision-making process was described as beginning with information received from the ambulance call centre, including, in some cases, the labelling of a patient's need as 'assist only'. In these cases, many interviewees reported that this formed a picture of a person who had not sustained any injury, but the majority recognised that the person may have experienced an injury or be medically unwell and that care was needed so as to not automatically assume the person was fit to be left at home. Only a minority considered that their assessment or care would differ from any other case:

"To me an 'assist only' is someone's fallen on the floor, you don't do any obs [vital signs], you put them back to bed and that's it." (A28)

Decision making also appeared to be influenced by the interviewees' definitions of a 'fall'. Some conformity with the study's definition of a fall (see table 1) was expressed and there was some agreement that a 'fall' was a significant event, usually if associated with a medical cause (such as loss of consciousness) and required assessment in the ED. However, about half of the interviewees also used terms such as a 'simple fall' or 'trip', and these events were described as less concerning than a 'fall', more likely to be attributed to external causes and considered less likely to need treatment in an ED:

"my personal understanding would be... hit by something or a medical condition has caused this person to collapse, as opposed to a trip or a stumble or just loss of balance." (C128)

Initial contact: assessing the situation for any immediate action required and establishing a rapport

The interviewees all articulated their main concern as identifying those older people for whom immediate conveyance to the ED was described as necessitated by one or more of the following: the patient's level of consciousness, pain, injuries associated with the fall or a long lie on the floor, or the patient's medical condition.

“'Do you have pain anywhere?' Address that first especially chest pain." (D207)

All interviewees spoke of making an immediate assessment as they entered the premises to establish the details surrounding the fall: what happened, when, and the possible cause for the fall.

Implicit to many of the interviewees' descriptions was the idea of taking care with the way in which questions were 
phrased, with attempts to establish a rapport being more explicitly described by a small number as a way through which to begin an assessment of the patient and gain accurate information about the circumstances of the fall:

“Well you say 'what are you doing down there?' Just to break the ice. It usually causes a laugh and then I find out how they managed to get down on the floor." (C125)

Rapport was also described as serving to gain the older person's trust so that later advice to convey to the ED would not be easily dismissed:

\begin{abstract}
"You get a lot of that (refusing to go to hospital) and when they say that straight away we say 'We're not worrying about that at the moment, let's make sure you're okay, let's find out why you're on the floor'." (C145)
\end{abstract}

\section{Continuing assessment: gathering and assimilating medical and social information}

In the absence of any clear immediate medical need to convey a patient to the $\mathrm{ED}$, all the interviewees described a further assessment used to make a decision about whether to convey the patient or not. Most interviewees described this assessment as comprising: the patient's medical history, medications, current condition and mobility; and the social and environmental circumstances.

\section{"So with an elderly person you check all their medications as well to see if they could lead into what illnesses they do have. So if they've got blood pressure, if they have pleurisy, or heart problems." (D207)}

This information was variously reported as being gained from the older person themselves, from professional and non-professional carers, and from any records kept in the patient's home, although a small number of interviewees considered that the latter were not able to provide the information on medical history, which would have been useful. Some also noted that acquisition of information from patients could be affected by the patient's anxiety regarding the possibility of having to be conveyed to the $\mathrm{ED}$, or by their poor health or cognitive impairment:

\section{"...they just don't know because some of them are on so many pills and you say 'Why are you on these tablets?' And they say 'I don't know' or 'because the doctor told me to go on them' and we don't know, unless it's written down." (A28)}

In terms of the patient's current condition, the vast majority of interviewees described their assessment of 'vital signs' as that of recording blood pressure, respiratory rate, pulse rate and level of consciousness, and adhering to the standard requirement for ambulance staff of obtaining two readings of 'vital signs' while they are with the patient (on arrival and then either before leaving the patient's home or handing over to the ED).

\footnotetext{
"...I used to do just one (set of vital signs) but now I do two, just to see if there's any change since I've been on. Maybe 10 min apart or 5 min apart and their pulse rate and their blood pressure and their sats [oxygen saturation level]." (A22)
}

The majority of interviewees described such observations as a necessary part of patient care and as providing a baseline in assessing the patient's condition. However, some variation in the types (some performing electrocardiograms (ECGs), while some just pulse and blood pressure checks) and number of observations the interviewees performed was reported. This part of the assessment appeared to be affected by the home circumstances. For the majority this assessment was related to the patient's presenting condition, although two interviewees suggested a reluctance to carry in heavy diagnostic equipment if access to the home was not straightforward.

Most interviewees spoke of the importance they assigned to an assessment of the patient's mobility in establishing if the patient was safe to be left at home, in particular attempting to ascertain if any change in mobility or pain had resulted from the fall. However, difficulties were again noted in assessment in this group of frail patients, for whom no baseline information was available:

\section{"So you're asking them does it hurt anyway. 'Yes my knees really hurt'. 'Is that how they are normally?' 'Yes' 'So there's nothing new?" (C119)}

"You would always test mobility. You always say to them 'If you can't walk then we'll take you in because you can't get around your own flat'. So we always make sure that they can walk around the flat." (D203)

Finally in this stage of assessment, eight of the interviewees spoke explicitly of the need to assess the patient's social and environmental situation given the complexity of factors they recognised as potentially causing a fall. These interviewees generally stated that they assessed the patient's living conditions to provide an indication as to how the patient was being cared for either by themselves or others. This could include checking who might be visiting the patient next and when, as well as assessing food, heating and environmental hazards:

"Sometimes you go into just death traps basically, and they have rolls of carpet everywhere and boxes lying about which obviously for an unstable old person is not that helpful. So you might move a few of them out of the way and say to them 'Have you had an assessment of your living circumstances?' sort of thing." (D207)

It was recognised that poor social and environmental circumstances did not necessarily indicate a need for conveyance to the ED:

"There may not be anything like illness or injury wrong with them in that way, so A\&Es [the ED] pointless. They're just going to sit there for $4 \mathrm{~h}$ and be sent home because as far as I know, it's the GP that refers them to the social services, and not really the A\&E department." (C145)

For half of those interviewed the presence of carers was an important factor in the decision whether to convey or not, with the absence of carers given as a reason in itself to convey the patient in some cases.

\section{Making a conveyance decision: a process of negotiation, referral and professional defence}

Interviewees were asked about their use of the CAT, which had been evaluated in the quantitative stage of the study. All bar one of those who reported having completed the CAT stated this to have been performed retrospectively, after a decision about conveyance had been made and the patient care episode completed. The participants appeared to be mostly ambivalent about the value of the standardised assessment tool as a decision-making instrument, all stating that they considered that it documented an assessment process that they already followed. In fact the interviewees described their knowledge from prior work experience as being most influential in the decisions they made, with 'instinct' named as a factor that was given credence in the assessment process. 
"It's a job you learn through experience and not by knowledge on paper really I feel." (A22)

"You walk in and you get that feeling. I don't like the look of this." (D203)

That said, nine of the interviewees made positive statements about the tool in terms of the extra information provided by themselves for the patient's GP as part of the CAT.

Interviewees' responses suggested that there are some situations where they felt it was clear what decision needed to be made, while in others, where the patient's needs were complex or where the patient refused, the decision was more anxiety provoking. Clear decisions to leave a patient at home were described as usually indicated when the fall was assessed to have been an explainable accidental slip or trip and all concerned agreed that there was no reason for conveyance:

\begin{abstract}
"They've just clipped the door frame or just tripped over a bit of carpet. We just put the carpet back where it's supposed, tell them it's an easy thing to get sorted out with the warden, and then that's it. There's no need for the GP to go round, they've only tripped on a bit of carpet." (D203)
\end{abstract}

That said, three participants suggested that they always try to convey the patient to the ED whatever the perceived cause of the fall due to concern about what will happen next for the older person. This approach was reflected for a larger number of interviewees in situations where the complexity of the patient's health and social care situation meant that the only solution to addressing some of the patient's perceived needs was seen to be conveyance to the ED. This was described by half the interviewees as facilitating further assessment of the patient with a view to implementing increased community care support.

All interviewees reported that the process of gaining a patient's agreement to go to the ED was not always straightforward, with some patients reluctant to go in:

\section{"You've just got to be a bit careful with elderly people, as I say they don't like going into hospital much. So they'll try and do everything to get out of it." (D207)}

Interviewees reported a range of reasons why older people, in their experience, refuse to go to the ED following a fall. Most of the interviewees reported sympathy with the older person's concerns and respect for their right to decide not to go to the ED, but all participants described their attempts to counter the older person's fears, using words such as 'negotiate', 'cajole', 'bribe', or 'coax' to describe the process they entered into. If this period of negotiation failed then firmer tactics were described:

"And then I feel like I've got to bully them into going, and it is a horrible situation to be in because sometimes there's nothing wrong with them. They've accidentally tripped over their carpet, they've not actually injured themselves. They've got an underlying illness but I don't know their history. So because of that I have to take them in, because I have to cover myself..." (A22)

This quote also highlights another issue that was clearly of importance to the interviewees, that is, 'covering your back'. The vast majority of interviewees spoke of anxiety associated with decisions, particularly concerns about potential repercussions arising from the risk associated with non-conveyance to the ED and strategies to mitigate this perceived risk:

"Everyone has got the right to refuse to go to hospital....So in that circumstance I would try contacting either a relative or doctor to come round and see them and use their persuasion as well. If that failed, then the only thing we could do is to advise the patient or relatives if they get any worse we will call back. And to record what we've done.... and contact a doctor in some way." (A8)

However, the experiences participants recounted in contacting GPs were varied, with access difficulties predominant (all bar one reported issues at times) and a lack of feedback on the outcome of their referrals explicitly reported as frustrating by three participants. Referral to other services, such as district nursing or social services was also mentioned by a small number of participants. Although these referrals could be problematic, one example of a positive conclusion was also given:

\begin{abstract}
"She was being left at home. We found a GP, he came out and visited her...the GP was fuming that she'd been sent home, and we were here, so I got him to 'phone Social Services as well. And the GP's driver, he fixed her burner so she then had heating and hot water." (A22)
\end{abstract}

Another method that a smaller number of interviewees described to 'cover their backs' was asking the patient to sign to say they had refused conveyance, although there was doubt expressed whether this would be a defence should there be any future medical issue. Three participants also described the CAT as a way to mitigate risk, although one other suggested that the CAT (and the research project) were in fact another form of scrutiny causing concern about repercussions and therefore encouraging conveyance to the ED.

\section{DISCUSSION}

The descriptions of the assessment of older people who fall by the 12 interviewees showed some similarity and some diversity of views. Analysis showed the described decision-making process to be complex and instinctive as well as reliant upon prior knowledge, and impacted upon by a number of external factors. The ambulance staff who were interviewed had all been part of an intervention study that introduced and tested the safety of a CAT to increase the consistency of these assessments and decisions with older people who had fallen. However, interviewees generally were clear that they did not use the CAT as an aid to patient assessment and the subsequent decision making, even though the CAT had been completed (retrospectively) in some of the cases they attended in the implementation period. This statement, and the diversity of views expressed, indicates that the intervention that was designed to formalise processes did not have a uniform impact on ambulance staff and their reported assessment practices. It is possible that the study training and focus on falls at the interviewees' work bases had impacted upon their processes in practice, although this is unclear in the absence of any qualitative data from ambulance staff prior to implementation of the CAT and was not the focus of this study.

At the core of the described decision-making process there appeared to be a tension between two models of decision making: a formal one based on procedures, and an informal one based on experience and intuition. The formal process was put in place by the ambulance service, in the case of the established procedures advocating conveyance for all to the $\mathrm{ED}$, and the new procedure (CAT and referral to the GP) being evaluated, which prompted decision making as to whether the ED was appropriate or not. The informal process of decision making appeared to be based upon custom and practice at the level of frontline ambulance staff. Both of these processes were described by study participants, with an emphasis on what they saw as the 'downside' (the need to 'cover their backs' when not conveying a patient) of the informal processes. Although this finding was 
similar to those of other studies on non-conveyance ${ }^{15}$ it is nevertheless perhaps surprising considering that the interviewees had all recently been through training to introduce new formal processes that recognised, and were in fact designed, to support the existing informal processes. The new processes gave organisational 'permission' for ambulance staff to use their knowledge and experience in decision making with regard to conveyance to the ED. At the same time, it is recognised that the CAT introduced a new formal process in presenting an expectation that an assessment was fully documented to support the decision and the patient's GP was informed if non-conveyance resulted from the assessment, and the majority of our participants completed an assessment tool for only some of the cases they attended.

These two types of decision making, formal and informal, have been discussed in the literature. The 'formal' approach has been described in terms of a rational hypotheticodeductive model; by contrast, the 'informal' approach has traditionally been identified with clinical decision making based on intuition and experience. The decision-making style is now considered to be related to the case and the individual clinician. ${ }^{18}$ It is not considered possible to completely eliminate the role of intuition, which involves personal decision rules, ${ }^{19}$ and which is attributed to conditions of uncertainty. ${ }^{20}$ Such conditions of uncertainty are considered to be particularly present in emergency situations where there are many 'unknowns' in patient assessment, resulting in the use of heuristics (rules of thumb) and a reliance on previous experience. ${ }^{21} 22$ However, intuition has been widely considered to be a source of error and bias. ${ }^{23}$

It is possible that the appearance of both types of decision making in this study reflects the understandable dilemma that the interviewees described-they are responsible for dealing with the emergency situation of an older person having just fallen, while also being faced with complex chronic health and social situations that, in the absence of other alternatives, leaves no other option than to take the patient to the ED. In addition ambulance staff reported needing to respond to the patient's wishes regarding conveyance. In fact the interviewees were clearly sympathetic to the older person who wishes to remain at home, and it is this context that makes the low usage of the CAT more difficult to interpret. The two, apparently conflicting findings do contribute to the somewhat confused picture we see from interviewees of a fall being considered routine and easy to deal with (ie, something they can be 'certain' about), and as a real cause for concern in their practice (perhaps due to the uncertainty present). In this situation it is also understandable why other factors, such as the patient's reluctance to be conveyed or the presence of carers, also impact upon the decision. $^{12}$

The interviews also highlighted that ambulance staff consider themselves to be on the periphery of community care provision. Links to specific falls services in local areas, such as that reported in a trial of a paramedic practitioner role ${ }^{24}$ may serve to alleviate such issues. It is not known if such a model would be generalisable to all ambulance staff, such as those who participated in our study, who showed concern about 'covering their backs', despite the interviews taking place after a study intervention that had sanctioned non-conveyance if the "clinical assessment tool' indicated this was appropriate.

\section{Study limitations}

This is a small study, but there was a convergence of views around the process involved in the assessment of older people who fall. A convenience sampling approach was used, and women, EMT rather than paramedic staff, and those with few years experience are slightly over-represented in the participants. The EMTs and paramedics of whatever previous length of experience taking part in this study received the same training in use of the assessment tool specific to this study. Prior to this study neither EMTs nor paramedics received training in the care of older people who fall or in decision making for nonconveyance. Our findings are also perhaps counterintuitive; although the use of set procedures has often been associated with novice staff and intuition with more experienced staff, ${ }^{25}$ some participants in our sample described the role of 'instinct', even though they were less experienced staff. The participants also reveal a cross-section of staff in terms of CAT usage-those who never, occasionally or frequently used the CAT-was achieved.

\section{Conclusions}

This small qualitative study has presented a description of an assessment process that highlights the complexity of making decisions about whether or not to convey older people who fall and present to the emergency ambulance service; a predominance of informal decision-making processes; and a reluctance to use a systematic decision-making tool. However, descriptions of falls and the factors that influence decisions point to the need for a consistent message and support for ambulance staff in this area. This in itself generates a significant challenge to those with education roles in the ambulance service. Further research is needed to look at how new care pathways that offer an alternative to the ED may influence decision making around nonconveyance, and thus patient health outcomes.

Acknowledgements We acknowledge the contribution of the study participants, of Fiona Ross and Christopher Tye in commenting on early drafts, and of the peer reviewers whose comments and questions enabled us to improved the manuscript.

Funding The study was funded by the Department of Health Research Programme to Support Implementation of the National Service Framework for Older People. The funding body did not contribute to the study design, to the collection, analysis and interpretation of the data or to the writing of the manuscript.

\section{Competing interests None.}

Ethics approval This study was conducted with the approval of the Metropolitan Multi-Centre Research Ethics Committee.

Contributors $\mathrm{MH}$ designed (with $\mathrm{HS}$ and $\mathrm{JC}$ ) and coordinated the study, analysed and interpreted the data and drafted the manuscript (with SV). SV analysed and interpreted the data and drafted the manuscript. HS designed the study, participated in its coordination and helped draft the manuscript. AP contributed to interpretation of results and provided structure to the presentation of the analysis. JC conceived of and designed the study, and participated in its coordination. FM participated in coordination of the study. SP recruited the participants, carried out the interviews and participated in analysis of the data. All authors contributed revisions to the paper, and read and approved the final manuscript.

Provenance and peer review Not commissioned; externally peer reviewed.

\section{REFERENCES}

1. Bergeron $\mathbf{E}$, Clement J, Lavoie $\mathrm{A}$, et al. A simple fall in the elderly: not so simple. J Trauma 2006;60:268-73.

2. Sikron F, Giveon A, Aharonson-Daniel L, et al. My home is my castle! Or is it? Hospitalizations following home injury in Israel, 1997-2001 [see comment]. Isr Med Assoc J 2004;6:332-5.

3. Kellogg International Work Group. The prevention of falls in later life. A report of the Kellogg International Work Group on the Prevention of Falls by the Elderly. Dan Med Bull 1987;34(Suppl 4):1-24.

4. Tinetti ME. Factors associated with serious injury during falls by ambulatory nursing home residents. J Am Geriatr Soc 1987:35:644-8.

5. Scuffham P, Chaplin S, Legood R. Incidence and costs of unintentional falls in older people in the United Kingdom. J Epidemiol Community Health 2003:57:740-4.

6. Department of Health. National Service Framework for Older People. London: Department of Health, 2001. 
7. National Collaborating Centre for Nursing and Supportive Care. The assessment and prevention of falls in older people. London (UK): National Institute for Clinical Excellence, 2004.

8. Weiss SJ, Chong R, Ong M, et al. Emergency medical services screening of elderly falls in the home. Prehosp Emerg Care 2003; 7:79-84.

9. Snooks H, Halter M, Close J, et al. Emergency care of older people who fall: a missed opportunity. Qual Saf Health Care 2006;15:390-2.

10. Snooks H, Porter A, Gaze S, et al. Non-conveyance of 999 patients and related clinical documentation: Policy, practice and views (Report). Swansea: CHIRAL, School of Medicine, Swansea University, 2004.

11. Marks PJ, Daniel TD, Afolabi O, et al. Emergency (999) calls to the ambulance service that do not result in the patient being transported to hospital: an epidemiological study. Emerg Med J 2002;19:449-52.

12. Close JC, Halter M, Elrick A, et al. Falls in the older population: a pilot study to assess those attended by London ambulance service but not taken to A\&E. Age Ageing 2002;31:488-9.

13. Porter A, Snooks H, Whitfield R, et al. 'Should I stay or should I go? Deciding whether to go to hospital after a 999 call'. J Health Serv Res Policy 2007;12(Suppl 1):32-8

14. Snooks H, Kearsley N, Dale J, et al. Towards primary care for non-serious 999 callers: results of a controlled study of "Treat and Refer" protocols for ambulance crews. Qual Saf Health Care 2004;13:435-43.

15. Porter A, Snooks H, Youren A, et al. "Covering our backs": ambulance crews attitudes towards clinical documentation when emergency (999) patients are not conveyed to hospital. Emerg Med J 2008;25:292-5.
16. Halter M, Close JCT, Snooks $\mathrm{H}$, et al. Fit to be left: can ambulance staff use an assessment tool to decide if an older person who has fallen can be safely left at home? Final Report to Department of Health Research Programme to Support Implementation of the National Service Framework for Older People, 2005. http:// www.healthcare.ac.uk/research/documents/fit-to-be-left-report.pdf (accessed May 2009)

17. Popay J, Mallinson S, Kowarzik U, et al. Developing public health work in local health systems. Prim Health Care Res Dev 2004;5:338-50.

18. Evans C. Clinical decision making theories: patient assessment in A\&E. Emerg Nurse 2005;13:16-19.

19. Hall KH. Reviewing intuitive decision-making and uncertainty: the implications for medical education. Med Educ 2002;36:216-24.

20. Beresford EB. Uncertainty and the shaping of medical decisions. Hastings Cent Rep 1991;21:6-11

21. Cioffi J. A study of the use of past experiences in clinical decision making in emergency situations. Int J Nurs Stud 2001;38:591-9.

22. Croskerry P. Achieving quality in clinical decision making: cognitive strategies and detection of bias. Acad Emerg Med 2002;9:1184-204.

23. Tversky A, Kahneman D. Judgment under uncertainty: heuristics and biases. Science 1974:185:1124-31.

24. Mason S, Wardrope J, Perrin J. Developing a community paramedic practitioner intermediate care support scheme for older people with minor conditions. Emerg Med J 2003:20:196-8.

25. Benner P. From novice to expert: excellence and power in clinical nursing practice. Menlo Park: Addison-Wesley, 1984.

\section{Images in emergency medicine}

\section{Asymptomatic common carotid artery dissection caused by blunt injury}

A 16-year-old boy was referred to our hospital by a general practitioner on suspicion of damage to the right carotid artery. He hit the right side of his neck against a door during basketball and lost consciousness for about $10 \mathrm{~s}$. The physical examination revealed no abnormalities except an abrasion and bruise on the right side of the neck. Ultrasonography showed the presence of a flap and severe stenosis within the right common carotid artery. A three-dimensional CT angiography showed severe dissection of the right common carotid artery with aneurysmal formation on the proximal side of the dissection (figure 1A,B). Additional angiography also demonstrated the same findings. After antiplatelet therapy for 10 days, we performed balloon-expandable stent deployment for the severe stenosis because of deterioration of the stenosis on ultrasonography. The postoperative course was uneventful and he was discharged on the third day.
Traumatic dissection of the carotid artery is a rare condition, which accounts for $<1 \%$ of all patients with blunt injuries. It is an important factor for young people because it could cause strokes. A three-dimensional CT angiography can demonstrate the presence of the dissection less invasively, and the extent of the dissection is recognised with ease.

\section{N Fukunaga, M Hanaoka, K Sato}

Department of Neurosurgery, Tokushima Red Cross Hospital, Irinoguchi, Komtsushima-cho, Komatsushima-shi, Tokushima, Japan

Correspondence to Naoto Fukunaga, Department of Cardiovascular Surgery, Kobe City Medical Center General Hospital, 4-6 Minatojimanakamachi, Chuo-ku, Kobe Hyogo 650-0046, Japan; naotowakimachi@hotmail.co.jp

\section{Competing interests None.}

Patient consent Obtained.

Provenance and peer review Not commissioned; not externally peer reviewed

Accepted 5 February 2009

Published Online First 6 October 2010

Emerg Med J 2011;28:50. doi:10.1136/emj.2009.073288
Figure $1(A, B)$ Three-dimensional CT angiography is shown. The arrow indicates the dissection of the right common carotid artery. Severe stenosis and aneurysmal formation are recognised.
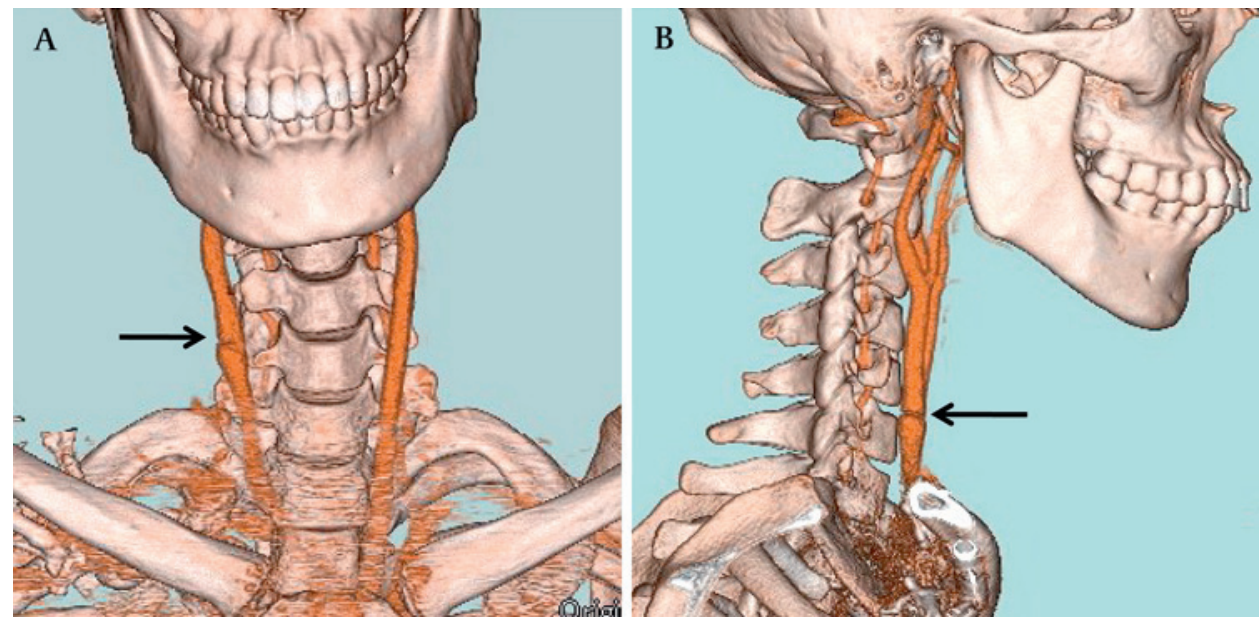